\title{
LIMIT SECTIONS AND UNIVERSAL POINTS OF CONVEX SURFACES
}

\section{Z. A. MELZAK}

1. The following question, raised originally by S. Mazur, appears in S. M. Ulam's collection of mathematical problems [1]: does there exist a closed convex surface whose plane sections give all plane closed convex curves, up to affinities? While this problem is apparently still unsolved the answer is almost certainly negative. At least three different extensions of the problem could be considered: (1) to allow the plane sections an equivalence up to a larger class, or perhaps a larger group, of transformations than the affinities, (2) to ask that the set of all plane sections of the surface should only contain a sufficiently large subset of the set of all closed convex curves, for instance, the set of all convex polygons of given diameter or perimeter, all analytic ovals of fixed length, or all ovals of given constant width, and (3) to generalize the concept of a plane section.

This note is concerned with the last possibility. Instead of plane sections of a surface one considers its limit sections. Roughly speaking, these are limits of sequences of magnified sections of a surface by sequences of planes converging to a supporting plane. For instance, if a strictly convex surface is sufficiently regular the limit section will always be an ellipse with axes proportional to the square roots of the principal radii of curvature. Thus the limit section is a generalization of Dupin's indicatrix.

The following notation will be used: $C$ and $D$ will denote curves, other capital letters will usually denote surfaces, $P$ will be reserved for planes, small letters will stand for points, and small Greek letters will be non-negative constants. A surface (curve) will always mean a closed strictly convex surface (a closed plane convex curve). A part of a surface cut off by a plane will be called a cap. The set of all curves will be denoted by $\mathcal{u}$.

2. Let $S$ be a surface and let $O X Y Z$ be a Cartesian frame with the origin $O$ inside $S$. Let a sequence $\left\{P_{n}\right\}$ of planes converge to a supporting plane $P$ of $S$ at $s$. Suppose that all planes $P_{n}$ intersect $S$ and let $C_{n}=S \cap P_{n}$. Let $\left\{\lambda_{n}\right\}$ be a sequence of constants and let $\lambda_{n} C_{n}$ denote the curve similar to $C_{n}$ in the ratio $\lambda_{n}: 1$. The limit section $C$ of $S$ at $s$ with respect to the sequence $\left\{P_{n}\right\}$ is defined as the limit,

Presented to the Society August 28, 1958; received by the editors October 14, 1957. 
if it exists, of the sequence $\left\{\lambda_{n} C_{n}\right\}$. More accurately, it is the limit of suitable reorientations in space, by rigid motions, of the curves of the sequence $\left\{\lambda_{n} C_{n}\right\}$. It must be emphasized that a limit section depends on the choice of the sequence of the intersecting planes. Further, if $C$ is a limit section, so is $\lambda C$. The set of all limit sections of a surface at a point $s$ on it will be denoted by $\mathcal{C}(s)$. The set of all parallel limit sections at $s$, that is, limit sections formed with respect to sequences of planes parallel to a supporting plane at $s$, will be denoted by $\mathfrak{C}_{p}(s)$. A point $s$ of a surface is called universal if $\mathfrak{e}(s)=\mathfrak{U}$, and it is called $p$-universal if $\mathfrak{e}_{p}(s)=\mathcal{U}$. A surface $S$ is universal if $\bigcup_{s \in S} \mathfrak{C}(s)=\mathfrak{u}$ and it is $p$-universal if $\bigcup_{s \in S} \mathfrak{C}_{p}(s)=\mathfrak{u}$.

3. Theorem 1. There exists a surface $S$ with these properties: (1) $S$ is of class $C^{\infty}$ except at one point $s$, (2) $S$ possesses a unique supporting plane at $s,(3) s$ is a p-universal point.

Select in $u$ a countable basis of analytic curves $\left\{C_{n}\right\}, n=1,2, \cdots$ Let $O X Y Z$ be a Cartesian coordinate frame, let $\left\{\alpha_{n}\right\}$ be an increasing convergent sequence with $\alpha_{0}=0$, and let $\left\{\mu_{n}\right\}$ be a decreasing sequence with $\mu_{0}=1$ and $\lim \mu_{n}=0$. These sequences will be determined in the process of construction. Let $C_{0}$ be the unit circle about the origin in the plane $z=\alpha_{0}$. In the plane $z=\alpha_{n}$ place the curve $\mu_{n} C_{n}$ so that the following conditions are satisfied: $\mu_{n} C_{n}$ encircles the $z$-axis, the projection of $\mu_{n+1} C_{n+1}$ onto the plane $z=\alpha_{n}$ lies within $\mu_{n} C_{n}$, and any cone with vertex on $\mu_{n+1} C_{n+1}$ and with $\mu_{n} C_{n}$ for directrix contains $\mu_{0} C_{0}, \cdots, \mu_{n-1} C_{n-1}$ in its interior. The sequences $\left\{\alpha_{n}\right\}$ and $\left\{\mu_{n}\right\}$ can always be found so that the above conditions are satisfied. It follows now that there exists a cap $T$ with these properties: $T$ is based on $C_{0}$, its intersection with the plane $z=\alpha_{n}$ is $\mu_{n} C_{n}$, and it is of class $C^{\infty}$ at all points except at the point $s$ with coordinates $\left(0,0, \lim \alpha_{n}\right)$. The last property calls for the standard technique which utilizes the functions like $f(x)=\exp \left(-1 / x^{2}\right)$. In addition, by making the sequence $\left\{\alpha_{n}\right\}$ converge fast enough the cap $T$ can be made to possess a unique supporting plane at $s$.

Now complete $T$ to a surface by closing it up with a hemisphere and modify this surface along the join to a surface $S$ which is of class $C^{\infty}$ everywhere except at $s$. The surface $S$ is easily shown to satisfy the conditions of the theorem: for any $C$ in $u$ there exists a subsequence $\left\{C_{n_{j}}\right\}$ whose limit is $C$; now the planes whose equations are $z=\alpha_{n_{j}}$, together with the sequence $\left\{\mu_{n_{i}}^{-1}\right\}$ of constants, determine the parallel limit section $C$ at $s$.

THEOREM 2. There exists a surface S every point of which is universal. 
It will be shown first that it suffices to construct $S$ so that the set of its $p$-universal points is dense on it. Let $S$ be such a surface and let $s$ be any point on it. By assumption, there exists a sequence $\left\{s_{n}\right\}$ of $p$-universal points on $S$, which converges to $s$. By definition, for each point $s_{n}$ there exists a convergent sequence $\left\{P_{m n}\right\}$ of planes, $m=1,2, \cdots$, which converges to a supporting plane at $s_{n}$, and which determines, together with a sequence $\left\{\lambda_{m n}\right\}$ of constants, a parallel limit section $C$ at $s_{n}$. Here $C$ is an arbitrary member of $\mathcal{u}$. Now it is easy to show that the diagonal sequence $\left\{P_{n n}\right\}$, together with $\left\{\lambda_{n n}\right\}$, determines a limit section $C$ at $s$.

The surface $S$ with a dense set of $p$-universal points will be constructed by the process of successive approximations. Let $O X Y Z$ be a Cartesian coordinate frame and let $S_{1}$ be the unit sphere about the origin. Let $\left\{H_{n}\right\}$ be a sequence of half-rays through $O$, which is dense in the set of all half-rays through $O$. Let $\left\{\beta_{n}\right\}$ be a sequence of small positive constants, to be determined later on. Let $s_{1}=S_{1} \cap H_{1}$ and let $v_{1}$ be the point on $O s_{1}$ whose distance from $s_{1}$ is $\beta_{1}$. Put a plane $P_{1}$ through $v_{1}$ at right angles to $O s_{1}$ and remove from $S_{1}$ the cap based on $P_{1}$. Let $D_{1}=S_{1} \cap P_{1}$ and complete the remainder of $S_{1}$ to a surface $S_{2}$ by placing over $D_{1}$ a cap $T_{1}$. This cap is constructed as in the proof of Theorem 1 but it is based on $D_{1}$. Also, let $T_{1}$ be such that $S_{2} \subset S_{1}$. Now repeat the same procedure on $S_{2}$, using $s_{2}=H_{2} \cap S_{2}, \beta_{2}, v_{2}, D_{2}, P_{2}$ and $T_{2}$ in place of $s_{1}, \beta_{1}, v_{1}, D_{1}, P_{1}$ and $T_{1}$. In this way one obtains a surface $S_{3} \subset S_{2}$. Let the process be continued. In the limit there results a surface $S$ which will be shown to possess a dense set of $p$ universal points for an appropriate choice of the sequence $\left\{\beta_{n}\right\}$.

By construction, each $T_{n}$ contains a $p$-universal point $p_{n}$. Therefore at the $n$th stage $S_{n}$ there are $n-1$ such points $p_{1}, \cdots, p_{n-1}$. The sequence $\left\{\beta_{n}\right\}$ is to be selected so that the following conditions hold: (1) during the deforming of $S_{n}$ into $S_{n+1}$ the points $p_{1}, \cdots, p_{n-1}$, are left in place together with some neighbourhoods (these of course shrink with increasing $n$ ), and

(2) in the limit the points $\left\{p_{n}\right\}, n=1,2, \cdots$, still function as $p$ universal points on $S$.

The first condition is easily met by making $\left\{\beta_{n}\right\}$ tend to zero fast enough. The second condition is less simple but it can also be satisfied in the same way.

At the $n$th stage $S_{n}$ let $P^{1}$ be the unique supporting plane of $S_{n}$, and also of $S$, at $p_{1}$. Let $P_{x 1}$ be the plane parallel to $P^{1}$ on the originside of it, and whose distance from $P^{1}$ is $x$. Let $C_{n x 1}=P_{x 1} \cap S_{n}$, let $f_{n 1}(x)$ be the length of $C_{n x 1}$, let $F_{n x 1}=C_{n x 1} \cap\left(S-\cap_{j=1}^{n} S_{j}\right)$, let $g_{n 1}(x)$ be the length of $F_{n x 1}$, and let $h_{n 1}(x)=g_{n 1}(x) / f_{n 1}(x)$. Roughly speaking, 
$h_{n 1}(x)$ is the percentage modification of a parallel section near $p_{1}$, due to the deformations of the first $n-1$ stages. The $p$-universal point $p_{1}$ on $S_{2}$ will also be $p$-universal on the limit surface $S$ if

$$
\lim _{x \rightarrow 0, n \rightarrow \infty} h_{n 1}(x)=0 .
$$

Now the deformation functions $h_{n k}(x)$ are formed for other $p$-universal points $p_{k}$, as they appear in the process. The condition (2) above will be satisfied by selecting $\left\{\beta_{n}\right\}$ so that $h_{n k}(x) \leqq x$. This completes the description of $S$ and the proof.

If $\varnothing$ is the empty set then trivially $\varnothing \subset \mathfrak{e}_{p}(s) \subset \mathfrak{e}(s) \subset \mathcal{U}$. The next theorem is concerned with the position of the two middle sets between the two extreme ones. It is shown that the most radical situation may occur.

Theorem 3. There exists a surface $S$ and a point $s$ on it, such that $\mathfrak{e}_{p}(s)=\varnothing$ and $\mathfrak{e}(s)=\mathcal{u}$.

Consider the basis $\left\{C_{n}\right\}$ used in the proof of Theorem 1. Choose $q_{n}$ on $C_{n}$ so that the radius of curvature of $C_{n}$ attains its maximum $\gamma_{n}$ at $q_{n}$; let also $\delta_{n}$ be its minimum. Let $O X Y Z$ be a Cartesian coordinate frame and let $P$ denote the $O X Y$ plane. Let $\left\{\lambda_{n}\right\}$ be a sequence of constants, decreasing steadily to 0 and with $\lambda_{1}=1$. Consider the sequence $\left\{\lambda_{n} C_{n}\right\}$ and place its members in $P$ as follows: all the points $q_{n}$ coincide with the origin $O, \lambda_{n} C_{n}$ lies on the positive $x$-side of the $y$-axis and is tangent to it at $O$, and $\lambda_{n+1} C_{n+1}$ is inside $\lambda_{n} C_{n}$. To satisfy the last condition let $0<\lambda_{n}<\lambda_{n-1} \delta_{n} / \gamma_{n}$. Let $P_{n}$ be a plane through the $y$-axis at an angle $\theta_{n}$ to $P$. The sequence $\left\{\theta_{n}\right\}$ is steadily decreasing to 0 and $\theta_{1}=\pi / 2$. Transfer $\lambda_{n} C_{n}$ from $P$ to $P_{n}$ by rotating it through an angle $\theta_{n}$ about the $y$-axis, and denote the result by $\lambda_{n} C_{n}^{\prime}$. Now select $\left\{\theta_{n}\right\}$ and $\left\{\lambda_{n}\right\}$ so that a surface $L$ can be constructed to satisfy these conditions: $L \cap P_{n}=\lambda_{n} C_{n}^{\prime}, L$ possesses at the origin the unique supporting plane $P$, and the intersection of $L$ with the $O X Z$-plane is a curve whose radius of curvature at 0 is either vanishing or infinite. Intersect $L$ with the $O Y Z$-plane and let $M$ be the cap based on $O Y Z$ and containing the curves $\lambda_{n} C_{n}^{\prime}$. Complete $M$ to a surface $S$ as follows. The intersection of $S$ and the plane $z=\alpha$, on the negative $x$-side, is an ellipse with axes $a(\alpha)$ and $b(\alpha)$. These ellipses are determined so that $a(\alpha) / b(\alpha)$ tends to 0 together with $\alpha$, and $P$ is the unique supporting plane of $S$ at the origin. Now the surface $S$ and the origin $s$ satisfy the conditions of the theorem: $s$ is universal by the construction of $M$, so that $\mathcal{e}(s)=\mathcal{U}$; on the other hand, the curvature conditions on $S$ imply that the plane sections 
of $S$ at $s$ possess two radii of curvature one of which (in the $O Y Z$ plane) is $\gamma_{1}$ while the other one (in the $O X Z$-plane) is either zero or infinite. This is easily seen to preclude the existence of any parallel limit section at $s$. Therefore $\mathfrak{e}_{p}(s)=\varnothing$.

4. The definitions of $\mathfrak{e}(s)$ and $\mathfrak{C}_{p}(s)$ suffer from the dependence of the limit sections on the sequences of intersecting planes. A point $s$ of a surface $S$ will be called ordinary if $\mathcal{C}_{p}(s)=\{\lambda C\}$ for a fixed $C$ in $\mathcal{u}$, that is, if the parallel limit sections at $s$ do not depend on the choice of the sequence of the intersecting planes. A surface is ordinary if all its points are ordinary.

THEOREM 4. There exists an ordinary surface with a universal point.

First it will be shown that for any $C_{n}$ in the previously used basis $\left\{C_{n}\right\}$ there exists a surface of class $C^{\infty}$ at all points except for one point $s$ for which $\mathcal{C}_{p}(s)=\left\{\lambda C_{n}\right\}$. Let $K$ be a cone with the directrix $C_{n}$. Transform $K$ into $N$ by a compression in the axial direction. Let the compression be sufficiently strong near the vertex of $K$ to send it into a point $s$ on $N$, at which $N$ possesses a unique supporting plane. Also, let $N$ be of class $C^{\infty}$ everywhere except at $s$. On $N$ take now any cap containing $s$ and complete it to a surface which is of the class $C^{\infty}$ everywhere except at $s$.

Let $S_{1}$ be the unit sphere about the origin and let $A$ be an arc of a great circle, terminating at the north pole $s$ of $S_{1}$. On $A$ let $\left\{s_{n}\right\}$ be a sequence of points tending steadily to $s$. About $s_{n}$ as centre draw on $S_{1}$ a small circle $D_{n}$ of radius $\rho_{n}$. The radii are such that no two circles intersect. Let $V$ be the remainder of $S_{1}$ after the removal of the spherical caps based on all the $D_{n}$ 's. For the curve $C_{n}$ of the previously used basis construct a cap $V_{n}$ based on $D_{n}$ and of class $C^{\infty}$ everywhere except for one point $v_{n}$ for which $\mathcal{C}_{p}\left(v_{n}\right)=\left\{\lambda C_{n}\right\}$. As shown in the preceding paragraph, this can be done. Complete $V$ to a surface $S$ by placing $V_{n}$ over $D_{n}$ on $V$. Moreover, let $S$ be of class $C^{\infty}$ at all points except at the ordinary points $v_{n}$ and at their limit $s$. Let $h(x)$ be the deformation function at $s$ for the deformation of $S_{1}$ into $S$. It is defined in the same way as in the proof of Theorem 2. Now select the sequences $\left\{s_{n}\right\}$ and $\left\{\rho_{n}\right\}$ so that $h(x)$ tends to 0 together with $x$. The surface $S$ is ordinary at all points: at the sequence $\left\{v_{n}\right\}$ by construction, at $s$ by the condition on $h(x)$, and at all other points by the property of $C^{\infty}$. On the other hand, it easy to show that $s$ is a universal point.

5. Many other similar questions could be raised. Can the set $\mathfrak{C}(s)$ 
be empty? Does there exist an ordinary p-universal surface? What does the condition $\mathfrak{e}(s)=\{\lambda C\}, C$ fixed, imply? While for a surface of class $C^{2}$ a parallel limit section is an ellipse and for a surface of class $C^{3}$ any limit section is an ellipse, can a surface of class $C^{2}$ possess a nonelliptical limit section? What are the invariant properties, if any, of the set $\mathfrak{e}(s)$ and $\mathfrak{e}_{p}(s)$ under the change of $s$ on the surface, and under the transformations of the surface itself?

6. The author wishes to express his thanks to: the O.N.R. for a Research Associateship in Mathematics (1956-1957) held at the University of Michigan, the Department of Mathematics of the University of Michigan for its hospitality, and the referee and the Editor for thorough criticism and constructive suggestions.

\section{BiBLIOGRAPHY}

1. S. M. Ulam, Mathematical problems (mimeographed), Los Alamos, 1954.

University of Michigan AND

MCGILL UNIVERSITY 